\title{
Analyzing the Treatment Delivery Accuracy of the uRT Treatment Planning System and URT-Linac 506C by Use of AAPM TG 119 Test Cases
}

\section{DaZhen Jiang}

Wuhan University Zhongnan Hospital

\section{Dajiang Wang}

Wuhan University Zhongnan Hospital

Jiuling Shen

Wuhan University Zhongnan Hospital

Jun Zhang

Wuhan University Zhongnan Hospital

\section{Cheng Chen}

Wuhan University Zhongnan Hospital

\section{Zhirong Bao}

Wuhan University Zhongnan Hospital

\section{Hongli Zhao}

Wuhan University Zhongnan Hospital

Ji Chen

Wuhan University Zhongnan Hospital

Hui Liu

Wuhan University Zhongnan Hospital

Xiaoyong Wang ( $\square$ wangxiaoyong@znhospital.cn )

Zhongnan Hospital of Wuhan University

\section{Research}

Keywords: TG 119, Dosimetric Validation, Linac 506C, uRT_TPS, Flattening Filter (FF), Flattening Filter Free (FFF), Collapsed Cone Convolution algorithm

Posted Date: June 2nd, 2021

DOI: https://doi.org/10.21203/rs.3.rs-556651/v1

License: (c) (i) This work is licensed under a Creative Commons Attribution 4.0 International License. Read Full License 

Analyzing the treatment delivery accuracy of the uRT treatment planning system and uRT-Linac 506C by use of AAPM TG 119 test cases

Dazhen Jiang, Dajiang Wang, Jiuling Shen, Jun Zhang, Cheng Chen, Zhirong Bao, Hong li Zhao, Ji Chen , Hui Liu*, Xiaoyong Wang *

1. Department of Radiation and Medical Oncology, Hubei Key Laboratory of Tumor Biological Behaviors, Hubei Cancer Clinical Study Center, Zhongnan Hospital of Wuhan University, Wuhan, China, 430071

Corresponding authors contributed equally to this work:

Xiaoyong Wang; Department of Radiation Oncology, Zhongnan Hospital of Wuhan University, Wuhan 430071, China; E-mail: wangxiaoyong@znhospital.cn;

Hui Liu; Department of Radiation Oncology, Zhongnan Hospital of Wuhan University, Wuhan 430071, China; E-mail: hbzkznyy@ 163.com;

\begin{abstract}
:
Objective: The objective of this study was to investigate accuracy of the United Imaging Healthcare's uRT treatment planning system (uRT-TPS), by creating AAPM TG 119 test plans with respectively IMRT and VMAT techniques by homogeneous and heterogeneous phantom.

Materials and Methods: The plans were delivered to the homogeneous and heterogeneous phantom using the United Imaging Healthcare's uRT-Linac 506C. The overall dose calculation accuracy by uRT-TPS with Collapsed Cone Convolution (CC) and Monte Carlo (MC) algorithm was measured and analyzed by creating IMRT and VMAT plans for the 5 test geometries specified in TG 119, by using two kinds of beams FF photon beam and FFF photon beam. The point doses were measured with a Farmer type ion chamber and the fluences were measured with films respectively.
\end{abstract}

\title{
Results:
}

The result of position accuracy was shown that the worst position accuracy is $0.36 \mathrm{~mm}$ and the repeated positioning accuracy of MLC field location was less than $0.25 \mathrm{~mm}$. The symmetry deviation of MLC was less than $0.08 \mathrm{~mm}$. In this study, the CLs of sMLC, dMLC and VMAT plans with FF photon beams were $2.74 \%, 2.12 \%$, and $1.36 \%$ respectively. As for FFF photon beams, they were $3.76 \%, 2.14 \%$ and $2.90 \%$ respectively, whereas the counterpart CL specified in TG119 were $4.5 \%$ for the high dose regions and $4.7 \%$ for OAR regions. The CLs of Gamma Passing rates for sMLC, dMLC and VMAT plans were $4.59 \%, 5.35 \%$ and $2.15 \%$ for FF beam mode, and were $1.82 \%, 6.12 \%$ and $4.82 \%$ for FFF beam mode. For the heterogeneous phantom, the maximum deviation is $2.35 \%$ for $\mathrm{CC}$ and $2.63 \%$ for MC algorithm respectively.

\section{Conclusion:}

Based on this analysis which were performed in accordance with the TG 119 recommendations, it is evident that the URT treatment planning system and URT-Linac 506C have commissioned IMRT and VMAT techniques with adequate accuracy. and all uRT_TPS treatment plans were 
recognized as clinically acceptable.

\section{Keywords:}

TG 119; Dosimetric Validation; Linac 506C; uRT_TPS; Flattening Filter (FF); Flattening Filter Free (FFF) ; Collapsed Cone Convolution algorithm

\section{INTRODUCTION}

Intensity-modulated radiation therapy (IMRT) has replaced 3-dimensional conformal radiotherapy (3DCRT) because of its capacity to deliver a more conformal dose to the target and to spare normal structures. This helps the oncologist to escalate the dose to the tumor volume. It can improve the cure rate of cancer and better protect the organs at risk. A lot of researchers have reported that intensity-modulated radiation therapy has an advantage over three-dimensional conformal radiation therapy (3DCRT) ${ }^{[1,2]}$. IMRT plans can be delivered by use of either static multi-leaf collimator (sMLC) or dynamic multi-leaf collimator (dMLC) techniques ${ }^{[3]}$. Volumetric modulated arc therapy (VMAT) was introduced in radiotherapy with additional degrees of freedom to optimize the dose delivery ${ }^{[4]}$. During delivery of photon beams, VMAT varies the MLC aperture shape, dose rate and gantry speed. IMRT and VMAT allow delivery of highly conformal dose distributions to the tumor with reduced dose to surrounding normal tissue structures. These days IMRT and VMAT are very common treatment modalities throughout the world due to their clinical advantages for various anatomical sites ${ }^{[5]}$. There are several guidelines and protocols for IMRT and VMAT ${ }^{[6-8]}$. However, there is some evidence that IMRT and VMAT treatments may not always be as accurate as practitioners believe.

While the new techniques have improved treatment efficiency, they have also increased the complexity of treatment delivery which could potentially affect the accuracy. The Radiological Physics Center (RPC) published the results of 250 irradiation fractions of head and neck phantom and showed that 71 of the 250 fractions did not meet their basic accuracy requirements ${ }^{[12-11]}$. Thus, it is important to evaluate the planning accuracy in a more comprehensive manner, and set the baseline values during commissioning. There is a lack of commissioning studies that systematically verify that treatments can be planned and delivered accurately using IMRT and VMAT techniques for both FF and FFF photon beam modes. The American Association of Physicists in Medicine (AAPM) Task Group (TG) 119 has provided confidence limits (CLs) for IMRT commissioning of $6 \mathrm{X}$ mode with a set of test cases by combining data from nine participating institutions [12]. The established confidence limit can be used to determine the action level of patient-specific QA for IMRT treatment. In order to establish the tolerance limits, AAPM TG 119 defined the test cases and compared the results of multiple institutions. The TG 119 report baseline is also helpful to obtain confidence limits for new planning modalities like VMAT. Mynapati et.al ${ }^{[14]}$ published a scientific paper in the Journal of Applied Medical Physics to apply the AAPM TG 119 benchmark plans for VMAT. Nithya et al ${ }^{[15]}$ analyzed the performance of the planning system with VMAT technology by use of AAPM TG 119 test cases. Sharma et al [16] studied the VMAT commission for Versa HD linear accelerator using AAPM TG 119. In their 
study by following TG 119 guidelines, IMRT and VMAT plans were created, which assessed the basic capabilities of VMAT technique by comparison of VMAT and IMRT plans. After these studies, they made the conclusion of practicability of using TG 119 test cases for creating VMAT benchmark plans.

Along with the developments in dose-calculating algorithms, it is also equally important to use the QA phantoms which should have heterogeneity pattern inside same as in human body and the phantom should mimic the actual human body. Since calculating the dose accurately in a simple and homogeneous medium is easy for algorithm, it becomes tedious in a complex heterogeneous density medium. So the heterogeneous was used in this study.

The uRT-linac 506c medical linear accelerator (United imaging HealthCare co., LTD.) is an innovative type of accelerator which combines diagnostic helical CT with high dose rate intensity modulated accelerator to make it capable of performing precise radiotherapy with high resolution CT image guided, having the capability of adaptive radiotherapy, 4D image guided radiotherapy (IGRT) and so on. Since the diagnostic CT machine is sequentially located behind the accelerator, the couch stepping depth is remarkably longer than that of other counterparts, which demands a more rigid, sturdier and steadier couch compared to its peers. Its treatment planning system uRT-TPS can support various plan techniques, such as sIMRT, dIMRT, VMAT, 3D-CRT etc. The VMAT modality is quite similar to VMAT, which can vary continuously MLC aperture shape and radiation dose rate while the gantry is rotating at variable speed around patient's isocenter during treatment.

In this study, we want to validate the commissioning of uRT-Linac 506C and uRT-TPS by using AAPM TG 119 benchmark plans with respectively sIMRT, dIMRT and VMAT techniques. Before TG 119 test the performance of MLC was tested, like Wen et al.[16].Meanwhile, the photon beam modes were set respectively with FF and FFF. Since the uRT-Linac 506C is the world's first integrated CT-linac, this study will be an important technical report on this equipment.

\section{METHODS AND MATERIALS}

uRT-linac 506c medical accelerator

The uRT-linac 506c (United imaging HealthCare co., LTD., Shanghai) is a novel linear accelerator which combines diagnostic helical CT with high dose rate intensity modulated accelerator by locating a diagnostic CT behind the gantry sequentially on the same axis (see Figure 1). With this design, it would realize high quality image verification, adaptive radiotherapy, on-line CT simulation, treatment, and so on.

\section{MLC Position Accuracy}

Like Wen et al.[16], we first evaluate the performance of MLC.

1. Fully Open MLC leaves at gantry $0^{\circ}$ and collimator $0^{\circ}$. Use EPID to acquire the initial position $D_{0}$ of each leaf in image according to edge detection and record all MLC position provided by control system as $\mathrm{P}_{0}$. 
2. Move MLC from the initial position to $-12.5 \mathrm{~cm}$ and the other side to $-7.5 \mathrm{~cm}$ which means the center of radiation field is at $x=-10 \mathrm{~cm}$. Meanwhile, MLC moving direction from the initial position to other position is positive (+). Use EPID to acquire current position $D_{i}$ of each leaf in image and record all the MLC position provided by control system as $P_{i}$.

3. Move MLC to the same position with negative direction (-) and record different $D_{i}$ and $P_{i}$.

4. Repeat 2 3 steps using radiation field size in Table 1 and record all of the MLC position $D_{i}$ and $P_{i}$. Calculate the position accuracy at current gantry and collimator angle $P_{a i}$ with each MLC position with the formula below.

$$
P_{\text {ai }}=\left|\frac{\mu\left(D_{i}-D_{0}\right)}{m}-\frac{P_{i}-P_{0}}{q}\right|
$$

$\mu$ is resolution of EPID. $m$ is ratio between EPID height and ISO height. $q$ is ratio between MLC height and ISO height.

5. Repeat 2-4 steps for different sets of gantry and collimator angles listed in table1.

6. The maximum deviation of MLC position measuring at different gantry and collimator angle is assumed as the position accuracy $P_{a}$ :

$$
P_{a}=\max \left(P_{\text {ai }}\right)
$$

Table 1 Position accuracy experiment condition

\begin{tabular}{ccccc}
\hline \multicolumn{2}{c}{ Angle } & Move & field size & field center $\mathrm{x} / \mathrm{cm}$ \\
Gantry & Collimator & direction & $\mathrm{cm} \times \mathrm{cm}$ & \\
\hline $0^{\circ}$ & $0^{\circ}$ & + +-direction & $5 \times \mathrm{Y}_{\max }$ & \\
$90^{\circ}$ & $0^{\circ}$ & + +-direction & $5 \times \mathrm{Y}_{\max }$ & $-10 \mathrm{~cm},-5 \mathrm{~cm}, 0 \mathrm{~cm}, 5 \mathrm{~cm}, 10 \mathrm{~cm}$ \\
$180^{\circ}$ & $90^{\circ}$ & + +-direction & $5 \times \mathrm{Y}_{\max }$ & \\
$270^{\circ}$ & $0^{\circ}$ & + +-direction & $5 \times \mathrm{Y}_{\max }$ & \\
\hline
\end{tabular}

Position accuracy should be less than $1 \mathrm{~mm}$ at treatment panel. The result is shown in Figure

1.The worst position accuracy is $0.37 \mathrm{~mm}$. 


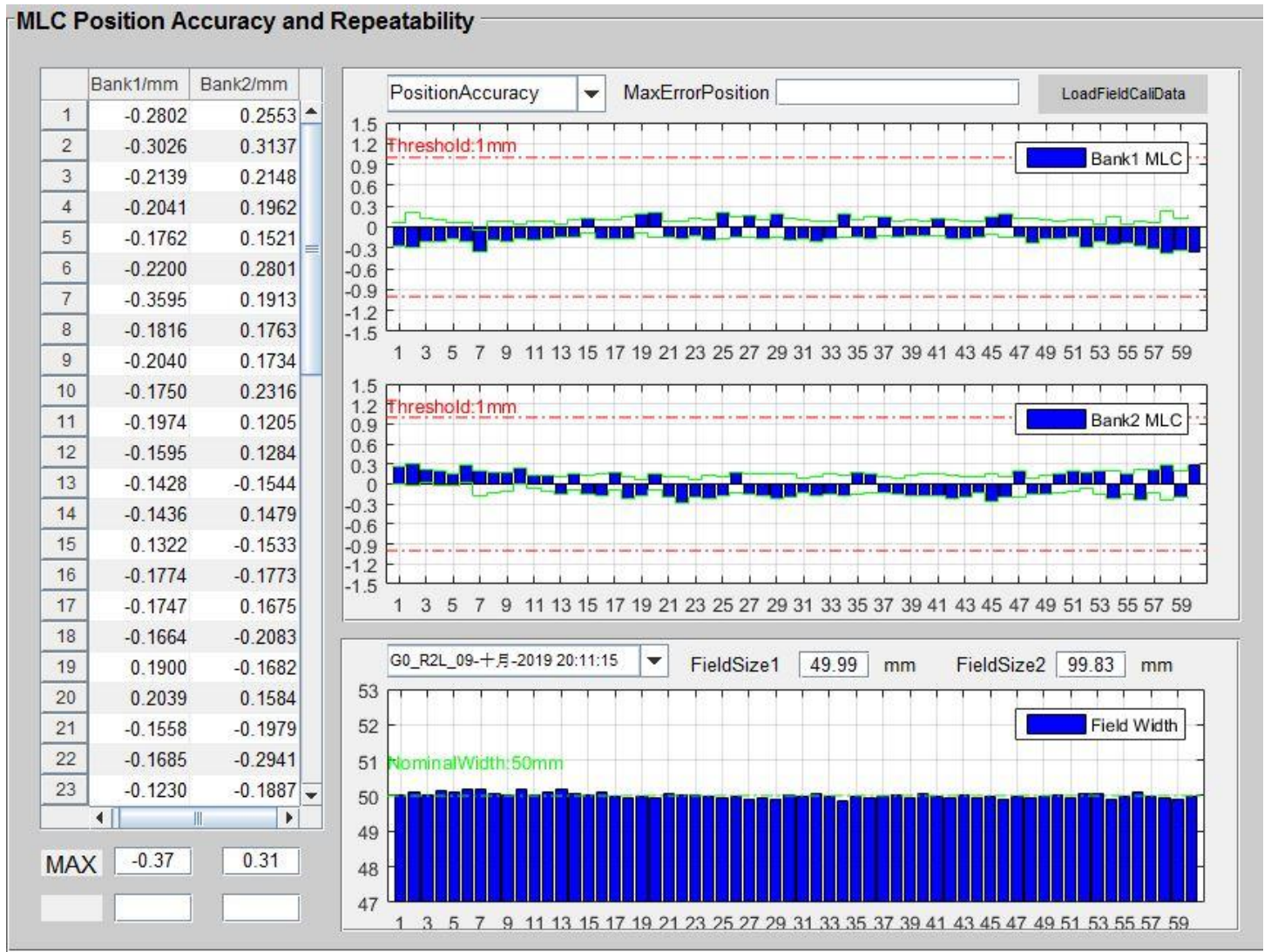

\section{MLC Position repeatability}

1. Move MLC with the maximum velocity and different direction to generate $5 \mathrm{~cm} \times$ Ymax cm filed size respectively with centers at $\mathrm{x}=-10 \mathrm{~cm},-5 \mathrm{~cm}, 0 \mathrm{~cm}, 5 \mathrm{~cm}, 10 \mathrm{~cm}$ at gantry $0^{\circ}$ and collimator $0^{\circ}$. Use EPID to acquire the position of each leaf and record MLC position $\mathrm{D}_{i}$ at each condition, for example with inverse moving direction. Position repeatability $R_{i}$ at this gantry angle is:

$$
R_{\tilde{i}}=\left|\frac{\max \left(\mu D_{i}\right)-\min \left(\mu D_{i}\right)}{m}\right|
$$

2. According to the test conditions given in Table 2, repeat step 1 for different sets of gantry and collimator angles. Then the accuracy of MLC field repositioning is calculated by the formula below:

$$
R_{a}=\max \left(R_{\tilde{i}}\right)
$$

Table 2 Position repeatability experiment conditions 


\begin{tabular}{|c|c|c|c|c|c|}
\hline \multicolumn{2}{|c|}{ Angle } & \multirow{2}{*}{$\begin{array}{l}\text { field size } \\
\mathrm{cm} \times \mathrm{cm}\end{array}$} & \multirow[t]{2}{*}{ field center $\mathrm{x} / \mathrm{cm}$} & \multirow{2}{*}{ velocity } & \multirow{2}{*}{ direction } \\
\hline Gantry & Collimator & & & & \\
\hline $0^{\circ}$ & $0^{\circ}$ & $5 \times Y_{\max }$ & & Max & $\begin{array}{l}\text { positive/negative } \\
\text { direction }\end{array}$ \\
\hline $90^{\circ}$ & $0^{\circ}$ & $5 \times Y_{\max }$ & $-10 \mathrm{~cm},-5 \mathrm{~cm}, 0 \mathrm{~cm}$ & Max & $\begin{array}{l}\text { positive/negative } \\
\text { direction }\end{array}$ \\
\hline $180^{\circ}$ & $90^{\circ}$ & $5 \times Y_{\max }$ & $5 \mathrm{~cm}, 10 \mathrm{~cm}$ & Max & $\begin{array}{c}\text { positive/negative } \\
\text { direction }\end{array}$ \\
\hline $270^{\circ}$ & $0^{\circ}$ & $5 \times Y_{\max }$ & & $\operatorname{Max}$ & $\begin{array}{l}\text { positive/negative } \\
\text { direction }\end{array}$ \\
\hline
\end{tabular}

The test results should be within the normal treatment distance where the repeated positioning accuracy of MLC field location should not be greater than $0.5 \mathrm{~mm}$. The result in this study was less than $0.25 \mathrm{~mm}$.

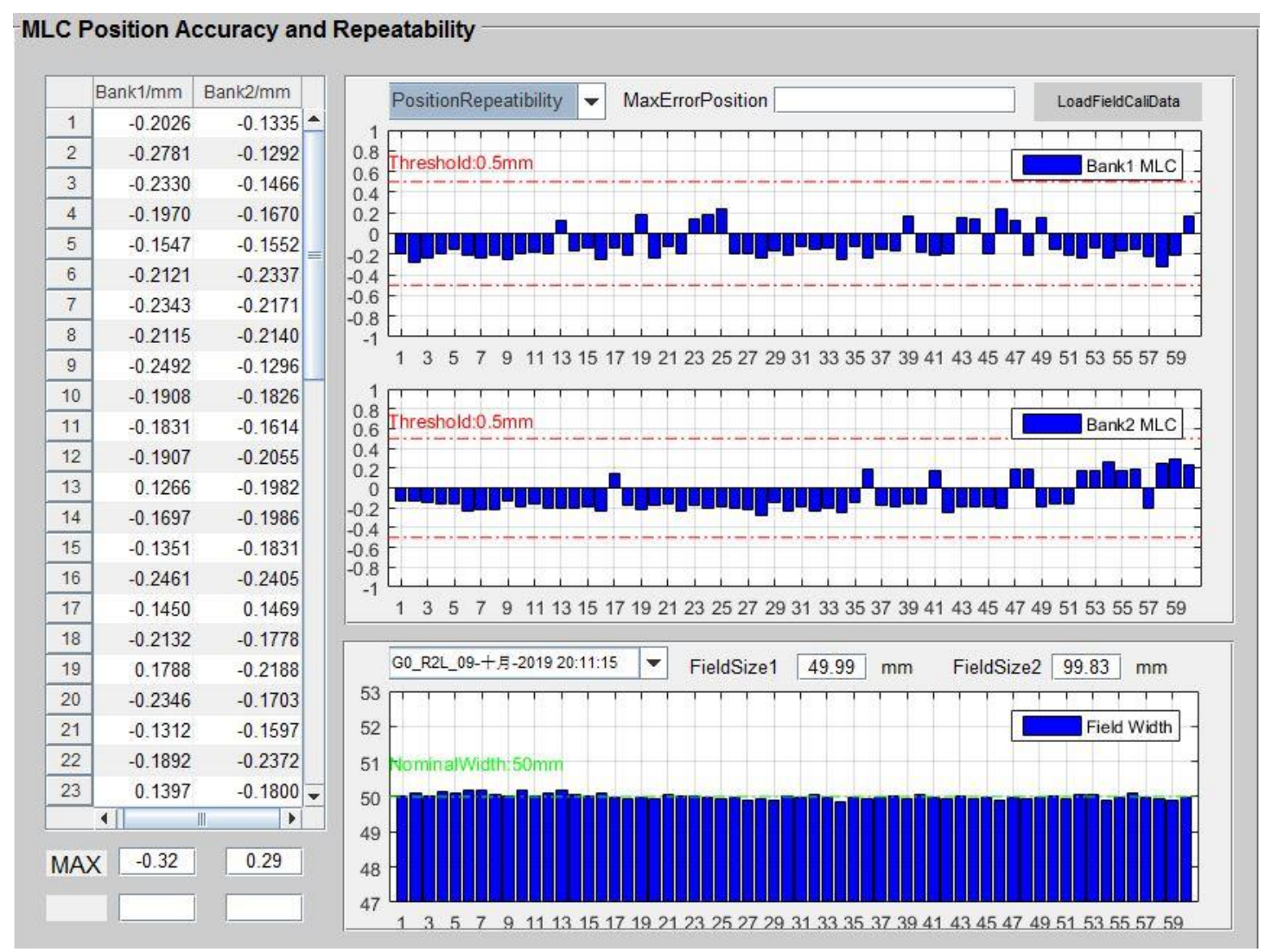

\section{Leaf Homogeneousity (Picket Fence)}

1. Under the test condition that the gantry angle and the collimator angle are both at $0^{\circ}$, the driving device makes all MLC move in the same direction to the positions of $-12.3 \mathrm{~cm}$ and $-11.7 \mathrm{~cm}$, namely, MLC forms the radiation field of $0.6 \mathrm{~cm} \times Y \max \mathrm{cm}$ where the center is at $\mathrm{x}=-12 \mathrm{~cm}$.

2. Based on the modeled relation between gray values measured by EPID and radiation 
dose, the dose distribution for each field was obtained. The distance $\Delta d_{i}$ between the maximum dose point of the dose distribution issued from each pair of leaves and the radiation field center was then calculated:

$$
\Delta d_{i}=\left|d_{i}-d_{0}\right|
$$

3. Repeat step 2) in line with the conditions in the table below: the radiation field is $6 \mathrm{~cm} \times$ Ymax $\mathrm{cm}$ whose centers are $\mathrm{x}=-12 \mathrm{~cm},-8 \mathrm{~cm},-4 \mathrm{~cm}, 0 \mathrm{~cm}, 4 \mathrm{~cm}, 8 \mathrm{~cm}$ and $12 \mathrm{~cm}$ respectively.

Table 3 Test Conditions of MLC Picket Fench

\begin{tabular}{cccc}
\hline Angle & $\begin{array}{c}\text { Beam } \\
\text { Limiting } \\
\text { System }\end{array}$ & $\begin{array}{c}\text { Radiation } \\
\text { Field } \\
\mathrm{cm} \times \mathrm{cm}\end{array}$ & Center Locations of Radiation Field $/ \mathrm{cm}$ \\
\hline $0^{\circ}$ & $0^{\circ}$ & $0.6 \times \mathrm{Y}_{\max }$ & \\
$90^{\circ}$ & $0^{\circ}$ & $0.6 \times \mathrm{Y}_{\max }$ & $-12 \mathrm{~cm},-8 \mathrm{~cm},-4 \mathrm{~cm}, 0 \mathrm{~cm}, 4 \mathrm{~cm}, 8 \mathrm{~cm}, 12 \mathrm{~cm}$ \\
$180^{\circ}$ & $0^{\circ}$ & $0.6 \times \mathrm{Y}_{\max }$ & \\
$270^{\circ}$ & $0^{\circ}$ & $0.6 \times \mathrm{Y}_{\max }$ & \\
\hline
\end{tabular}

4. Calculate the average deviation of maximum dose points relative to the center of the 7 radiation fields for each pair of leaves. Thereof the maximum value shall meet the requirements.

$$
\overline{\Delta \mathrm{d}}=\frac{1}{n} \sum_{i=1}^{n} \Delta d_{i}
$$

$\Delta d_{i}$ was the measured leaf position deviation at the $\mathrm{i}^{\text {th }}$ location

$\overline{\Delta d}$ was the average value

$\mathrm{n}$ was the number of measurements 


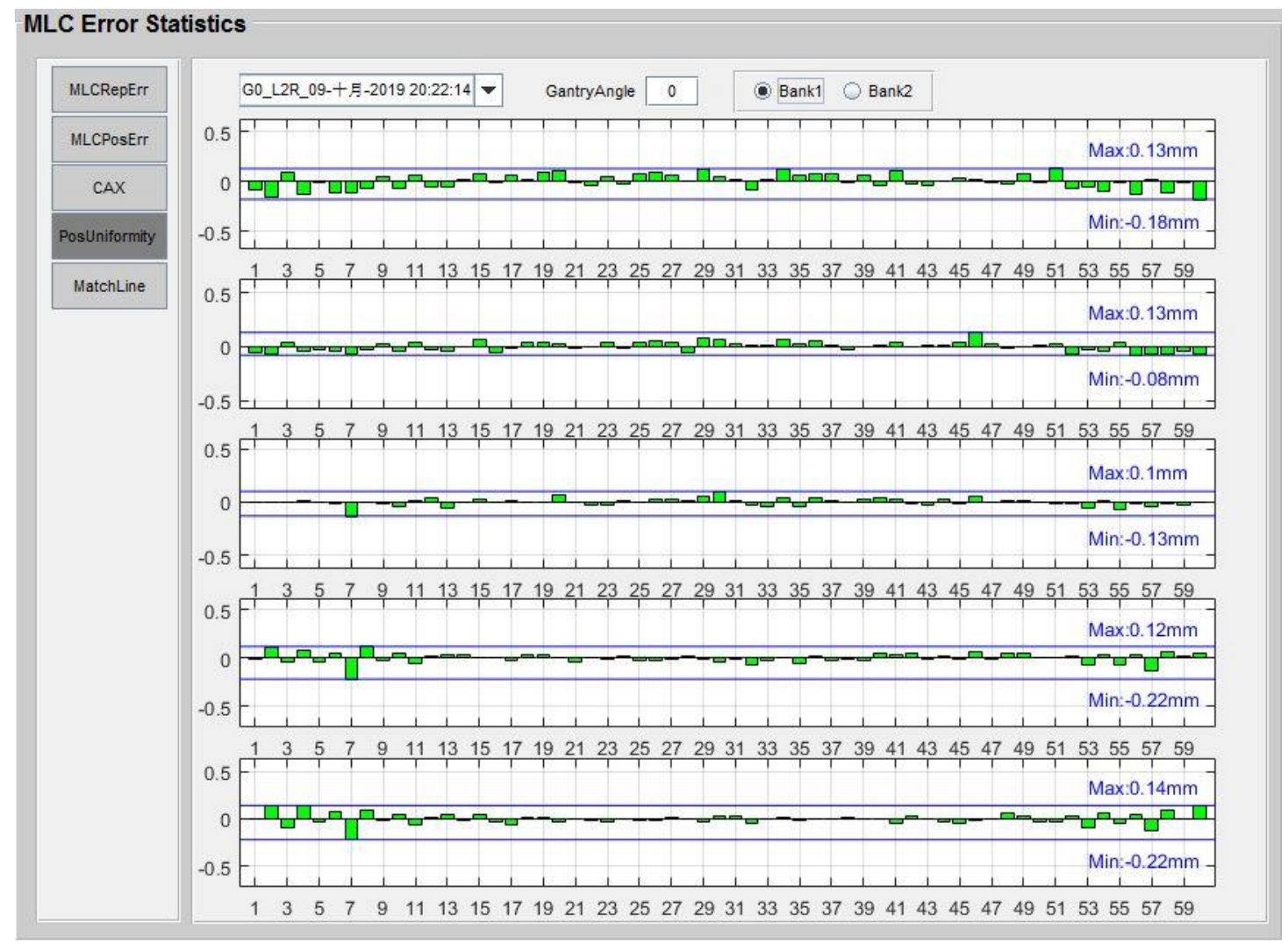

\section{Symmetry of MLC}

1. When the gantry angle is $0^{\circ}, \operatorname{MLC}(\mathrm{X}$ direction) and Jaw (Y direction) formed symmetrical fields $(10 \mathrm{~cm} \times 10 \mathrm{~cm})$.

2. The collimator system rotates at two angles $\left(0^{\circ}\right.$ and $\left.180^{\circ}\right)$ to compare the deviation to the field center. 


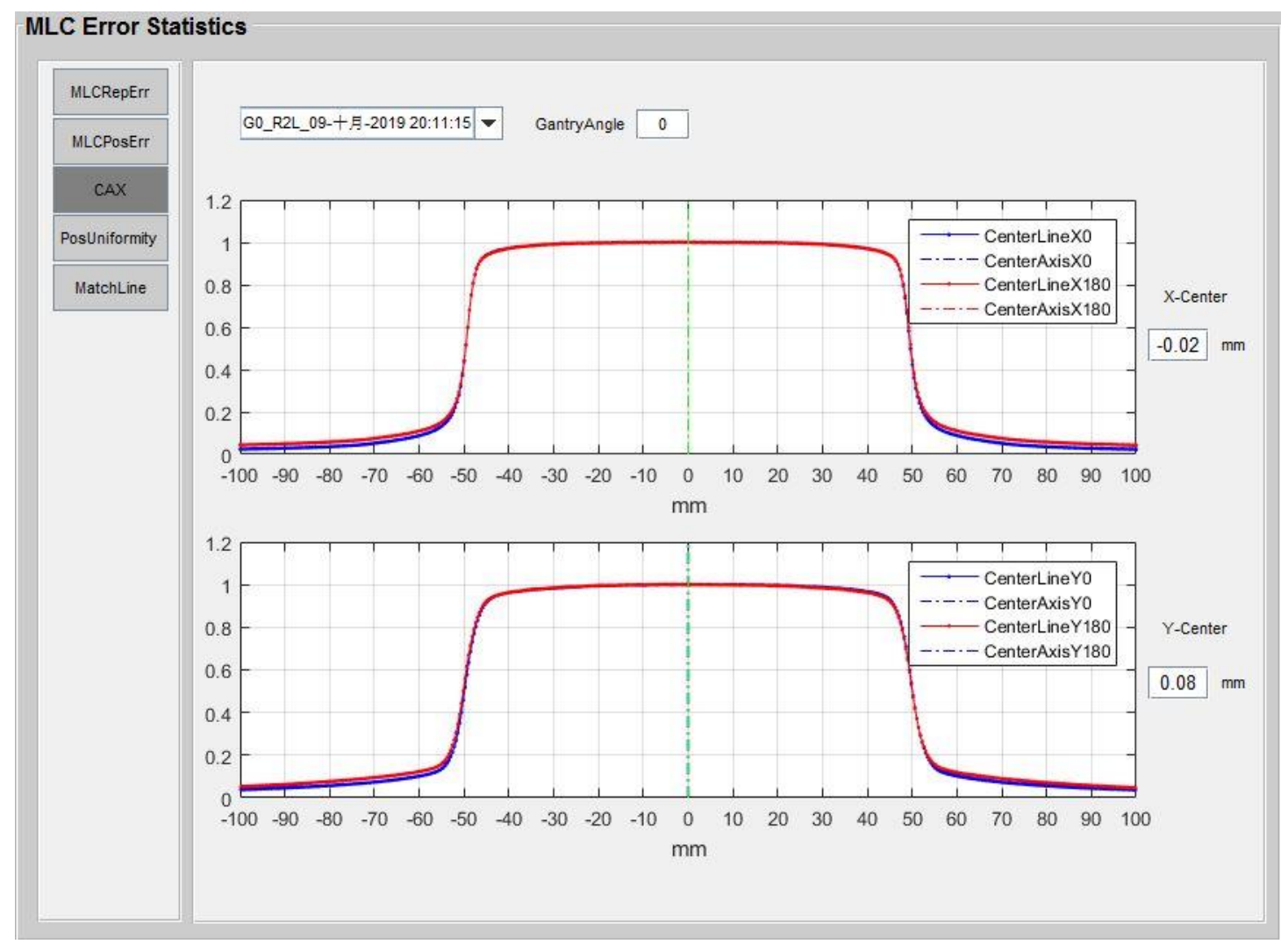

In this study, the symmetry deviation of MLC was less than $0.08 \mathrm{~mm}$.

\section{AAPM TG 119}

DICOM-RT images of computed tomography (CT) and structure sets were downloaded from http://www.aapm.org. and were transferred to the phantom in treatment planning system (TPS) to create verification plans for the measurement. The size of water equivalent slabs is $30 \mathrm{~cm} \mathrm{~L} \times 30$ $\mathrm{cm} \mathrm{W}^{\times} 15 \mathrm{~cm} \mathrm{H}$. We arranged the solid water blocks in different combinations to make sure the ion chamber measurements could be done at any depth specified in the AAPM TG 119 report. Four structure sets were created according to TG 119 report cases namely prostate, head-and-neck $(\mathrm{H}$ and N), Multi Target, C-shaped. AAPM TG 119 defines the beam arrangement, IMRT goals, and methods for analyzing the dosimetric results. For each test case, we generated three treatment plans (namely sIMRT, dIMRT and VMAT) on uRT-Linac 506C with 120 MLC (United Imaging Healthcare) in the uRT treatment planning system (clinical trial version), Fig 1. is a semi-dynamic VMAT technology of United Imaging Healthcare.

In order to compare the local results with TG119, we fully followed the methods and materials involved in the TG119(11). In the AAPM TG119, there are two primary tests, P1 and P2, which were used to evaluate the accuracy of planning and dosimetry systems. In the second primary test $\mathrm{P} 2$, We set asymmetric jaws which can create five bands of $3 \mathrm{~cm}$ width and the doses ranges from 40 cGy to $200 \mathrm{cGy}$.

For prostate and Multi Target cases, seven fields at $50^{\circ}$ intervals from the vertical $\left(0^{\circ}, 50^{\circ}\right.$, $100^{\circ}, 150^{\circ}, 310^{\circ}, 260^{\circ}$ and $\left.210^{\circ}\right)$ and one full arc $\left(179^{\circ}\right.$ to $181^{\circ}$ with a collimator angle $\left.30^{\circ}\right)$ were 
chosen for IMRT and VMAT plans respectively. For head-and-neck and C-shaped tests, nine fields at $40^{\circ}$ intervals from the vertical $\left(0^{\circ}, 40^{\circ}, 80^{\circ}, 120^{\circ}, 160^{\circ}, 320^{\circ}, 280^{\circ}, 240^{\circ}\right.$ and $\left.200^{\circ}\right)$ for IMRT and two complimentary full arcs were used for VMAT.

Fig 1: The linear accelerator of United Imaging Healthcare's CT linac uRT-Linac 506C

\section{Point dose measurement}

According to the AAPM TG 119, the IMRT and VMAT plans were transferred to the solid water phantom (figure 2) and the heterogeneous chest phantom (figure 3). The $0.125 \mathrm{cc}$ ionization chamber (PTW TM31010) was used to measure the point dose. The location of the ionization chamber must be considered, because the changes in the submillimeter level may significantly change the results.

The point dose measured by the ionization chamber is compared with the point dose calculated by the treatment plan system. According to the formula 1 , in the target area, the results of measurement error should be within $4.5 \%$, and in the Organs at Risk (OAR), the results of measurement error should be within $4.7 \%$,

$$
\text { discrepancy }=\frac{D_{\text {measured }}-D_{\text {calculated }}}{D_{\text {prescribed }}} \times 100 \%
$$

Where $D_{\text {measured }}$ (cGy), $D_{\text {calcutated }}(\mathrm{cGy})$ and $D_{\text {prescribed }}$ (cGy) are the measured, calculated, and prescribed doses, respectively.

\section{Fluence measurement}

GAFCHROMIC TM EBT3-1417 Films and EPSON Expression 11000XL Scanner and IBA OmniPro I"mRT 1.7 software (IBA Dosimetry Germany) were used for the gamma analysis of the composite dose distribution of the individual plans (both IMRT and VMAT) at different dose planes as specified in the AAPM TG 119 report.

Calibration films were irradiated for each photon energy with seven $5 \times 5 \mathrm{~cm} 2$ squares whose $\mathrm{MU}$ ranged from 0 to $1000 \mathrm{MU}$ (0MU, 50MU, 100MU, 200MU, 400MU, 800MU, 1000MU). The sampled optical density values of each color channel were then paired with the calculated dose values to establish the calibration curve through a cubic polynomial least squares fitting. The waiting time from irradiation to scanning was approximately 24 hours for postirradiation coloration. An Epson Expression 10000XL document flatbed scanner (Seiko Epson Corp, Nagano, Japan) with Epson Scansoftware was used to scan the films. Each film was scanned in the center of the scanner bed to allow for better scanner response homogeneousity. The films were scanned in transmission mode for better scanning stability with settings of 75 dot-per-inch and 48 bit RGB mode (16 bits per color channel). Images were exported in tagged image file format (TIFF) for 
analysis and image processing filters were disabled.

When scanning the films with EpsonTM expression 10000XL scanner, we kept all the films in the same direction. The scanned films were evaluated with OminiPro IMRT software by applying the gamma criteria of $3 \%$ dose difference and $3 \mathrm{~mm}$ DTA.

\section{Statistical analysis}

Statistical analyses were processed with SPSS ${ }^{\circledR}$ Statistics 19.0 software (IBM Corp., New York, NY; formerly SPSS Inc., Chicago, IL). A p-value less than 0.05 was considered as statistically significant.

\section{RESULTS}

Statistics for primary set planning results across all four Dicom structure sets are presented in Table 1-4. Each planner, in this study, had unique selections of planning parameters, but all plans followed the major guidelines, such as beam angles, isocenter point, dose per fraction, etc., as specified in TG 119.

\section{Preliminary tests P1 and P2}

The ion chamber measurements for primary test P1 and P2 are detailed in Table 1, the calculated doses were respectively $199.8 \mathrm{cGy}$ and $136.47 \mathrm{cGy}$, and the measured doses were respectively $201.06 \mathrm{cGy}$ and $137.58 \mathrm{cGy}$ with the deviation of $0.63 \%$ and $0.56 \%$

Table 1. The point dose measurement for test $\mathrm{P} 1$ and $\mathrm{P} 2$ at different bands for $6 \mathrm{MV}$

\begin{tabular}{lcccc}
\hline Test Location & Dose/Fraction & Measured dose(cGY) & Planned dose(cGy) & $\begin{array}{c}\text { (measured - } \\
\text { plan)/prescribed }\end{array}$ \\
\hline P1 Isocenter & 200 & 201.06 & 199.8 & $0.63 \%$ \\
P2 Isocenter & 200 & 137.58 & 136.47 & $0.56 \%$ \\
3 cm superior & 200 & 182.35 & 180.26 & $1.05 \%$ \\
3 cm inferior & 200 & 95.57 & 94.81 & $0.38 \%$ \\
6 cm superior & 200 & 217.96 & 216.65 & $0.66 \%$ \\
6 cm inferior & 200 & 51.68 & 51.46 & $0.11 \%$ \\
\hline
\end{tabular}

\section{Treatment plan statistics}


Figure 2 shows the test structures superimposed on a set of water-equivalent slab phantom. TG 119 problem set consists of four structure sets namely test Prostate, Multi Target, Head-and-neck ( $\mathrm{H}$ and $\mathrm{N}$ ), C-shaped (easy) and C-shaped (hard). All plan results for sMLC, dMLC \& VMAT plans achieved the planning goals except the D10 parameter of C-shaped (hard).

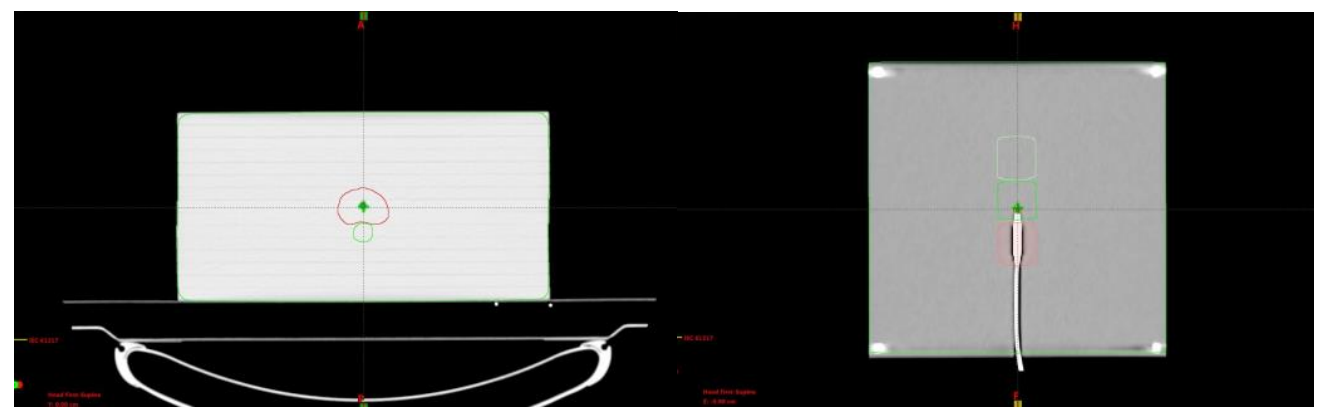

A

B

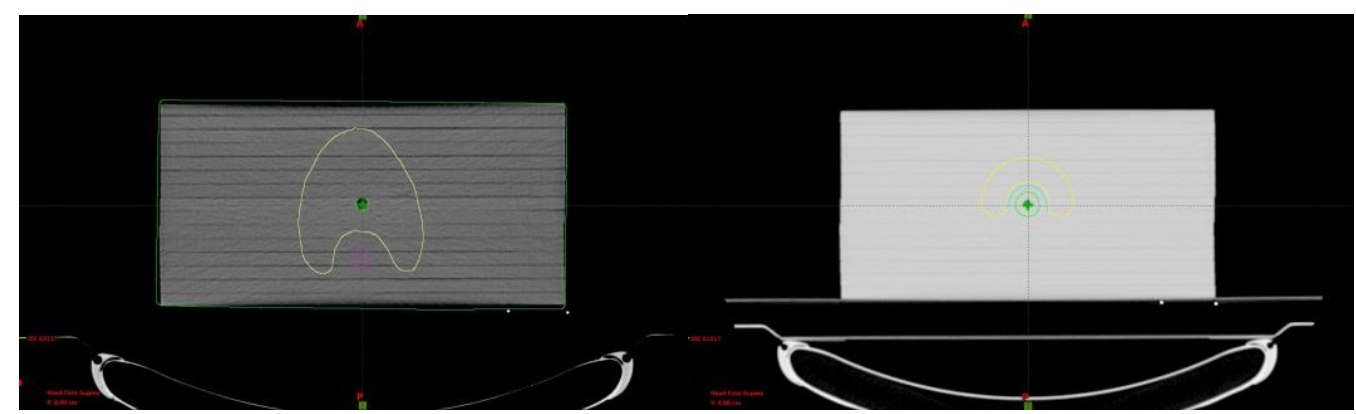

$\mathrm{C}$

$\mathrm{D}$

Figure 2. The test structures of Prostate (A), Multi Target (B), Head-and-neck (C) and C-shaped (D) by homogeneous phantom.

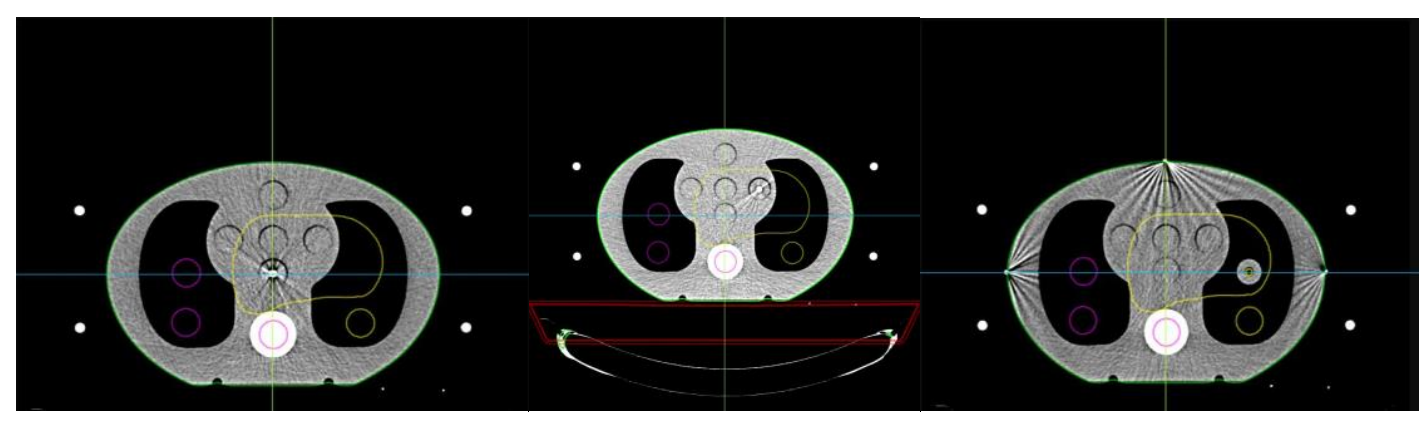

A

B

$\mathrm{C}$

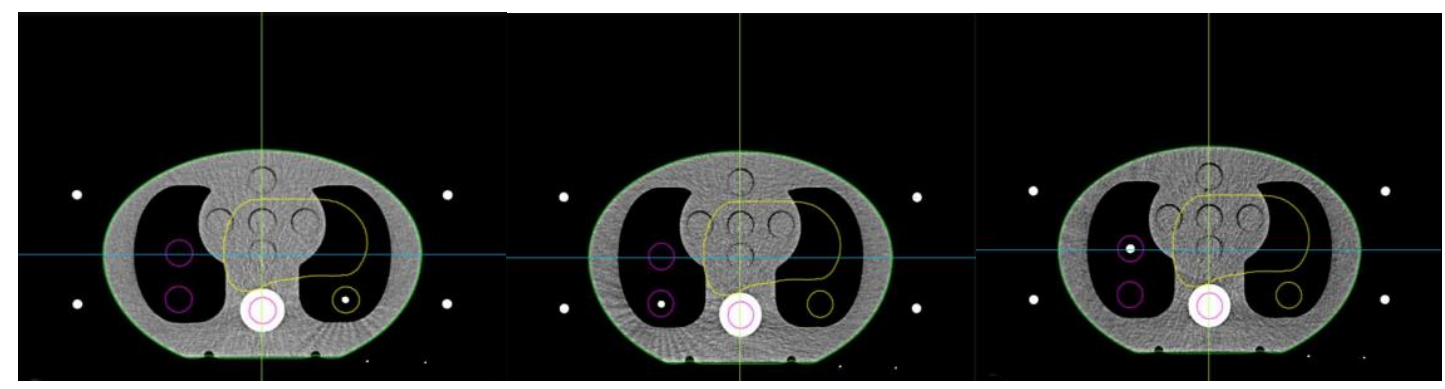


$\mathrm{C}$

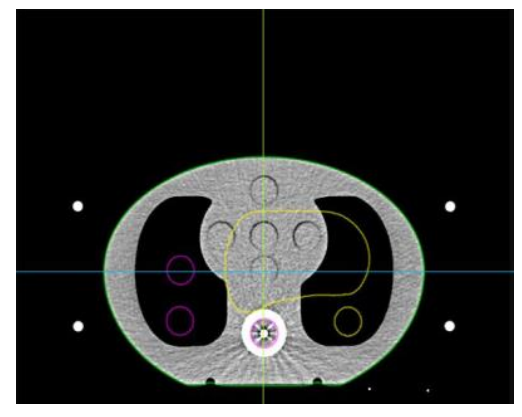

$\mathrm{F}$

Figure 3. The test structures of In PTV Down (A), In PTV Up (B), In PTV Lung (C), Out PTV Left (D), OUT PTV Right down (E), Out PTV Right Up (F) and In Spine (G) by heterogeneous chest phantom.

\section{Dosimetric parameters}

The dosimetric parameters of all treatment plans for Multi target, Prostate, Head and Neck, C shape Easy and C shape Hard are listed in Table 2. The planning criteria that we adopted here were in line with the Chinese regulation YY/T 0889-2013, which specifies functional performance and test methods of Intensity-modulated radiation planning system derived from TG119. To be noted, there is a difference between 0889 and TG119 protocol about C shape hard planning criteria: the 0889 used the dosimetry parameter Core D10 whereas the TG119 protocol used Core D5. All planning criteria required by 0889 have been achieved at our clinic for both FF and FFF beam modes.

Table 2. Dosimetric parameters of sMLC, dMLC and VMAT plans at respectively FF and FFF mode

FF

$\begin{array}{cccc}\text { Case } & \text { Locatio } & \text { Param } & \text { Goal } \\ n & \text { eter } & (\mathrm{cGy})\end{array}$

FF

FFF

\begin{tabular}{|c|c|c|c|c|c|c|c|c|c|}
\hline & & & & $\begin{array}{l}\text { sMLC } \\
\text { (cGy) }\end{array}$ & $\begin{array}{l}\text { dMLC } \\
\text { (cGy) }\end{array}$ & VMAT (cGy) & sMLC (cGy) & $\begin{array}{c}\text { dMLC } \\
(\mathrm{cGy})\end{array}$ & VMAT (cGy) \\
\hline & & D99 & $>5000$ & 5019.86 & 5019.91 & 5011.56 & 5055.73 & 5014.85 & 5001.22 \\
\hline Multi & Center & D10 & $<5300$ & 5259.89 & 5264.09 & 5215.26 & 5270.18 & 5296.18 & 5264.96 \\
\hline $\begin{array}{l}\text { Targe } \\
\text { t case }\end{array}$ & Superior & D99 & $>2500$ & 2616.11 & 2537.86 & 2621.99 & 2528.26 & 2594.16 & 2642.05 \\
\hline & & D10 & $<3500$ & 3494.82 & 3163.29 & 3343.43 & 3220.96 & 3385.79 & 3251.03 \\
\hline
\end{tabular}




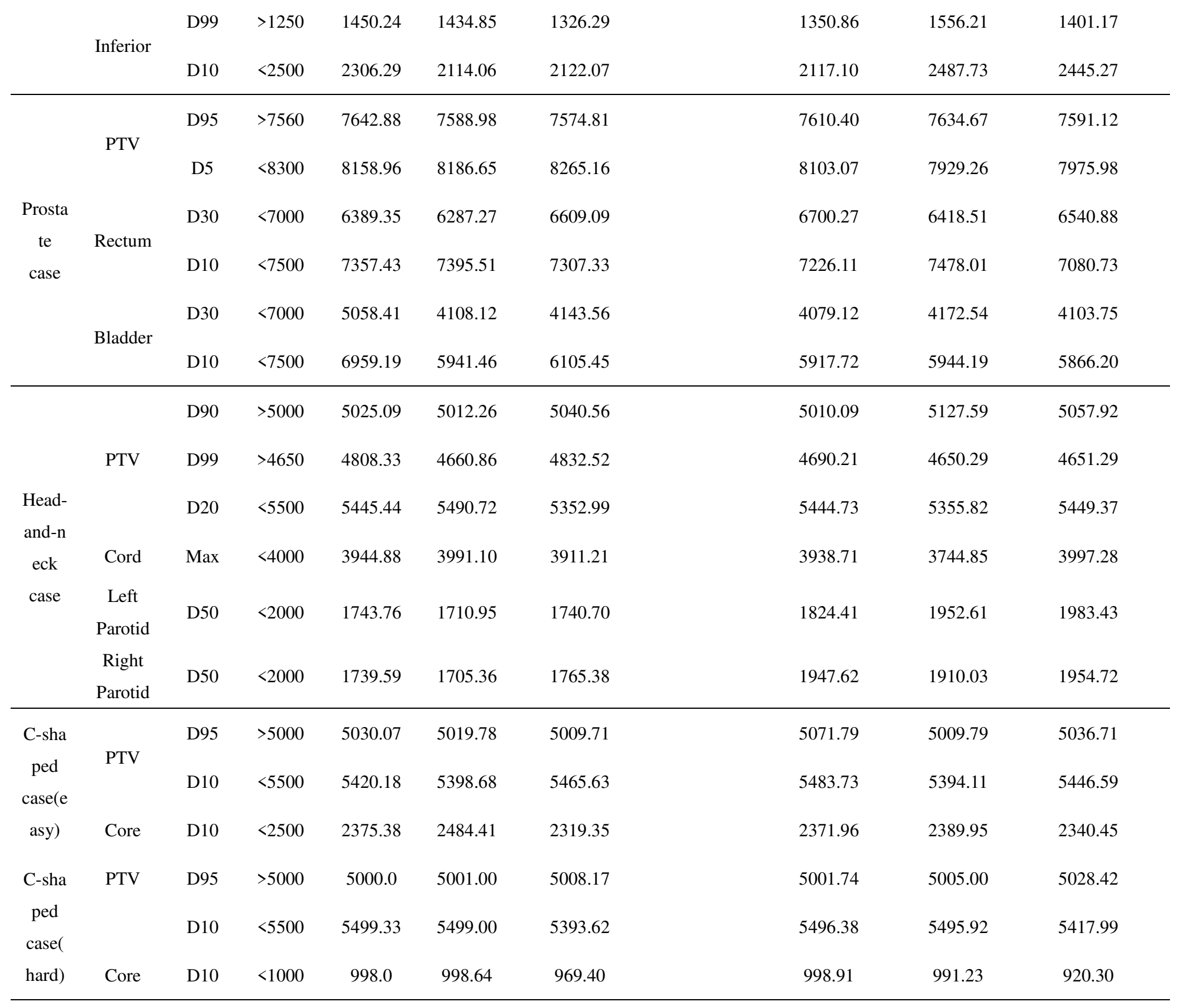

The point dose measurements of different test cases

Higher and lower point dose measurements (inside the target) are summarized in Table 3.

For FF plans, the mean discrepancies of sMLC, dMLC and VMAT were $-0.51 \%$, $0.32 \%$ and $0.40 \%$ respectively, and the standard deviations were $1.14 \%, 0.92 \%$ and $0.58 \%$ respectively, with the CL of $2.74 \%, 2.12 \%$ and $1.53 \%$ respectively. As for FFF plans, the mean discrepancies of sMLC, dMLC and VMAT were $-0.52 \%,-0.03 \%$ and $-0.70 \%$ respectively, and the standard deviations were $1.65 \%, 1.07 \%$ and $1.12 \%$ respectively, with the CL of $3.76 \%, 2.14 \%$ and $2.90 \%$ respectively. 
Table 3. Point Dosimetry Results in High and Low Dose Regions for sMLC, dMLC and VMAT plans with respectively FF and FFF mode by homogeneous phantom.

\begin{tabular}{|c|c|c|c|c|c|c|c|}
\hline \multirow[t]{2}{*}{ Case } & \multirow[t]{2}{*}{ Location } & \multicolumn{3}{|c|}{ FF } & \multicolumn{3}{|c|}{ FFF } \\
\hline & & sMLC & $\mathrm{dMLC}$ & VMAT & sMLC & $\mathrm{dMLC}$ & VMAT \\
\hline Multitarget & Isocenter & $-0.39 \%$ & $0.57 \%$ & $1.25 \%$ & $-1.57 \%$ & $0.27 \%$ & $-0.60 \%$ \\
\hline Multitarget & $\begin{array}{c}4 \mathrm{~cm} \text { superior to } \\
\text { isocenter }\end{array}$ & $-0.86 \%$ & $-0.82 \%$ & $0.71 \%$ & $-2.43 \%$ & $-0.88 \%$ & $0.21 \%$ \\
\hline Multitarget & $\begin{array}{c}4 \mathrm{~cm} \text { inferior to } \\
\text { isocenter }\end{array}$ & $-2.06 \%$ & $-1.39 \%$ & $-0.45 \%$ & $-1.74 \%$ & $-0.81 \%$ & $-3.66 \%$ \\
\hline Prostate & Isocenter & $-0.82 \%$ & $0.58 \%$ & $-0.11 \%$ & $-1.20 \%$ & $-0.41 \%$ & $-0.68 \%$ \\
\hline Prostate & $\begin{array}{l}2.5 \mathrm{~cm} \text { posterior } \\
\text { to isocente }\end{array}$ & $0.64 \%$ & $1.29 \%$ & $1.00 \%$ & $0.47 \%$ & $1.12 \%$ & $0.97 \%$ \\
\hline Head neck & Isocenter & $-1.20 \%$ & $-0.62 \%$ & $0.41 \%$ & $-2.94 \%$ & $-2.44 \%$ & $-0.79 \%$ \\
\hline Head neck & $\begin{array}{l}4 \mathrm{~cm} \text { posterior to } \\
\text { isocenter }\end{array}$ & $-1.67 \%$ & $0.87 \%$ & $0.00 \%$ & $-1.14 \%$ & $0.22 \%$ & $-0.51 \%$ \\
\hline $\begin{array}{l}\text { C-shaped } \\
\text { case(easy) }\end{array}$ & Isocenter & $0.39 \%$ & $0.99 \%$ & $0.90 \%$ & $2.17 \%$ & $1.16 \%$ & $-0.82 \%$ \\
\hline $\begin{array}{l}\text { C-shaped } \\
\text { case(easy) }\end{array}$ & $\begin{array}{l}2.5 \mathrm{~cm} \text { anterior } \\
\text { to isocenter }\end{array}$ & $-1.38 \%$ & $0.83 \%$ & $-0.13 \%$ & $0.78 \%$ & $-0.10 \%$ & $-0.49 \%$ \\
\hline $\begin{array}{l}\text { C-shaped } \\
\text { case(hard) }\end{array}$ & Isocenter & $1.18 \%$ & $0.92 \%$ & $-0.74 \%$ & $1.28 \%$ & $1.05 \%$ & $0.77 \%$ \\
\hline $\begin{array}{l}\text { C-shaped } \\
\text { case(hard) }\end{array}$ & $\begin{array}{l}2.5 \mathrm{~cm} \text { anterior } \\
\text { to isocenter }\end{array}$ & $0.72 \%$ & $0.22 \%$ & $0.52 \%$ & $0.56 \%$ & $0.43 \%$ & $0.38 \%$ \\
\hline & & $-0.51 \%$ & $0.32 \%$ & $0.40 \%$ & $-0.52 \%$ & $-0.03 \%$ & $-0.70 \%$ \\
\hline Stand & leviation & $1.14 \%$ & $0.92 \%$ & $0.58 \%$ & $1.65 \%$ & $1.07 \%$ & $1.12 \%$ \\
\hline Confidence li1 & $($ mean $) \pm 1.96 * \sigma$ & $2.74 \%$ & $2.12 \%$ & $1.53 \%$ & $3.76 \%$ & $2.14 \%$ & $2.90 \%$ \\
\hline
\end{tabular}

\section{Gamma analysis}


The planar dose of PTV in five cases was measured with Films recommended by TG119. The gamma analysis with criteria of 3\%/3mm was shown in Table 8. For the FF mode, the maximum gamma passing rate was $99.99 \%$ in head and neck and the minimum was $95.99 \%$ in prostate. The mean gamma passing rates of the four structures' plans with respectively technics sMLC, dMLC and VMAT were $98.40 \%, 97.67 \%$ and $99.38 \%$, with standard deviations of $1.53 \%, 1.54 \%$ and $0.78 \%$, and CL values were $4.59 \%, 5.35 \%$ and $2.15 \%$.

For the FFF, the maximum gamma passing rate was $100 \%$ in both head and neck sMLC and VMAT plans and the minimum was $95.31 \%$ in head and neck dMLC plan. The mean gamma passing rates of the four structures' plans with respectively technics SMLC, dMLC and VMAT were $99.32 \%, 97.01 \%$ and $99.02 \%$, with standard deviations of $0.58 \%, 1.60 \%$ and $1.96 \%$. The CL values were $1.82 \%, 6.12 \%$ and $4.82 \%$ respectively. The CL of FF and FFF photon beams were below 6.3. However, the value recommended by TG 119 was 12.4 .

Table 4. Gamma Analysis (3\%/3mm) Results of sMLC, dMLC and VMAT plans with respectively FF and FFF modes.

\begin{tabular}{|c|c|c|c|c|c|c|c|c|}
\hline & & $\mathrm{FF}$ & & & & FFF & & \\
\hline Case & Location & sMLC & $\mathrm{dMLC}$ & VMAT & sMLC & $\mathrm{dMLC}$ & VMAT & \\
\hline Multitarget & Isocenter & $98.44 \%$ & $97.08 \%$ & $98.08 \%$ & $99.83 \%$ & $98.56 \%$ & $95.52 \%$ & \\
\hline Prostate & Isocenter & $95.99 \%$ & $96.11 \%$ & $99.98 \%$ & $98.75 \%$ & $95.45 \%$ & $99.95 \%$ & \\
\hline Head neck & $\begin{array}{c}4 \mathrm{~cm} \\
\text { posterior to } \\
\text { isocenter }\end{array}$ & $99.76 \%$ & $99.84 \%$ & $99.99 \%$ & $\begin{array}{c}100.00 \\
\%\end{array}$ & $95.31 \%$ & $100.00 \%$ & \\
\hline $\begin{array}{l}\text { C-shaped } \\
\text { case(easy) }\end{array}$ & Isocenter & $98.14 \%$ & $96.66 \%$ & $99.38 \%$ & $98.78 \%$ & $97.16 \%$ & $99.89 \%$ & \\
\hline $\begin{array}{l}\text { C-shaped } \\
\text { case(hard) }\end{array}$ & Isocenter & $99.66 \%$ & $98.66 \% \quad 9$ & $99.46 \%$ & $99.23 \%$ & $98.58 \%$ & $99.75 \%$ & \\
\hline Mean & & $98.40 \%$ & $97.67 \%$ & $99.38 \%$ & $99.32 \%$ & $97.01 \%$ & $99.02 \%$ & \\
\hline $\begin{array}{l}\text { Standard } \\
\text { deviation }\end{array}$ & & $1.53 \%$ & $1.54 \%$ & $0.78 \%$ & & $0.58 \%$ & $1.60 \%$ & $1.96 \%$ \\
\hline $\mathrm{CL}=\mid$ & & & & & & & & \\
\hline $\begin{array}{c}100 \text {-mean } \mid+ \\
1.96 \sigma\end{array}$ & & $4.59 \%$ & $5.35 \%$ & $2.15 \%$ & & $1.82 \%$ & $6.12 \%$ & $4.82 \%$ \\
\hline
\end{tabular}

\section{Heterogeneous phantom Point dose measurement}

For the heterogeneous phantom test, the IMRT and VMAT plans were transferred to the heterogeneous chest phantom and the $0.125 \mathrm{cc}$ ionization chamber (PTW TM31010) was used to measure the point dose. When measuring the point dose, the location of the ionization chamber 
must be considered, because the changes in the submillimeter level may significantly change the results.

The point dose measured by the ionization chamber is compared with the point dose calculated by the treatment plan system according to the formula 1

Table 5. Point dose measurement results in heterogeneous phantom.

\begin{tabular}{|c|c|c|c|c|c|c|c|c|c|c|c|c|}
\hline \multirow[t]{3}{*}{ Location } & \multicolumn{6}{|c|}{$\mathrm{FF}$} & \multicolumn{6}{|c|}{ FFF } \\
\hline & \multicolumn{3}{|c|}{$\mathrm{CC}$} & \multicolumn{3}{|c|}{ MC } & \multicolumn{3}{|c|}{$\mathrm{CC}$} & \multicolumn{3}{|c|}{ MC } \\
\hline & sIMRT & dIMRT & VMAT & sIMRT & dIMRT & VMAT & sIMRT & dIMRT & VMAT & sIMRT & dIMRT & VMAT \\
\hline A & $0.70 \%$ & $1.55 \%$ & $1.15 \%$ & $0.49 \%$ & $-0.10 \%$ & $-0.52 \%$ & $-0.03 \%$ & $1.91 \%$ & $1.15 \%$ & $-0.63 \%$ & $-0.97 \%$ & $-1.54 \%$ \\
\hline B & $-0.73 \%$ & $-0.67 \%$ & $0.52 \%$ & $-2.45 \%$ & $-2.11 \%$ & $-0.61 \%$ & $-0.40 \%$ & $-0.58 \%$ & $0.52 \%$ & $-1.49 \%$ & $-2.07 \%$ & $-0.86 \%$ \\
\hline $\mathrm{C}$ & $1.43 \%$ & $-0.26 \%$ & $1.90 \%$ & $-0.74 \%$ & $-2.07 \%$ & $-1.32 \%$ & $0.25 \%$ & $-0.36 \%$ & $1.90 \%$ & $-2.26 \%$ & $-3.09 \%$ & $-1.54 \%$ \\
\hline D & $-0.07 \%$ & $-0.93 \%$ & $0.13 \%$ & $-1.27 \%$ & $-2.63 \%$ & $-2.33 \%$ & $0.20 \%$ & $-0.99 \%$ & $0.13 \%$ & $-1.18 \%$ & $-3.03 \%$ & $-1.24 \%$ \\
\hline E & $-2.35 \%$ & $-1.45 \%$ & $-0.31 \%$ & $-1.14 \%$ & $-0.55 \%$ & $-0.47 \%$ & $-0.79 \%$ & $0.09 \%$ & $-0.31 \%$ & $-0.95 \%$ & $-0.54 \%$ & $-0.38 \%$ \\
\hline $\mathrm{F}$ & $-2.34 \%$ & $-1.73 \%$ & $-0.91 \%$ & $-0.69 \%$ & $-0.29 \%$ & $-1.17 \%$ & $-0.71 \%$ & $-0.16 \%$ & $-0.91 \%$ & $-0.24 \%$ & $0.07 \%$ & $-0.99 \%$ \\
\hline G & $-1.10 \%$ & $-1.26 \%$ & $-0.46 \%$ & $-1.53 \%$ & $-1.02 \%$ & $-1.04 \%$ & $-0.03 \%$ & $-0.49 \%$ & $-0.46 \%$ & $-0.06 \%$ & $-0.92 \%$ & $0.39 \%$ \\
\hline Mean & $-0.63 \%$ & $-0.68 \%$ & $0.29 \%$ & $-1.05 \%$ & $-1.25 \%$ & $-1.07 \%$ & $-0.22 \%$ & $-0.08 \%$ & $0.29 \%$ & $-0.97 \%$ & $-1.51 \%$ & $-0.88 \%$ \\
\hline $\begin{array}{l}\text { Standard } \\
\text { deviation }\end{array}$ & $1.44 \%$ & $1.10 \%$ & $0.98 \%$ & $0.90 \%$ & $1.01 \%$ & $0.65 \%$ & $0.42 \%$ & $0.94 \%$ & $0.98 \%$ & $0.76 \%$ & $1.24 \%$ & $0.69 \%$ \\
\hline $\begin{array}{l}\text { Confiden } \\
\text { ce limit }= \\
(\text { mean }) \pm\end{array}$ & $3.46 \%$ & $2.83 \%$ & $2.21 \%$ & $2.81 \%$ & $3.23 \%$ & $2.34 \%$ & $1.04 \%$ & $1.93 \%$ & $2.21 \%$ & $2.46 \%$ & $3.94 \%$ & $2.24 \%$ \\
\hline $1.96 * \sigma$ & & & & & & & & & & & & \\
\hline
\end{tabular}

Note: the test structures of In PTV Down (A), In PTV Up (B), In PTV Lung (C), Out PTV Left (D), OUT PTV Right down (E), Out PTV Right Up (F) and In Spine (G) by heterogeneous chest phantom. 


\section{DISCUSSION}

The evaluation of MLC performance was performed based on the standard recommendations, including Picket Fence tests, delivered in both stationary and rotational gantry modes. The radiation pattern relative to that of the corresponding open fields was analyzed for different combinations of MLC position accuracy, MLC position repeatability, picket fence and symmetry of MLC. Rangel and Dunscombe[21] evaluated the dosimetric consequences of suboptimal MLC performances. They showed that a $0.3 \mathrm{~mm}$ systematic error leads to a $2 \%$ change in the equivalent uniform dose. The deviation could stem from various sources, including measurement uncertainty and slight differences in MLC leaf material/ design. Therefore, it is important to verify the systematic of MLC values. While the values in this study less than $0.02 \mathrm{~mm}$ in X center and less than 0.08 in $Y$ center.

In this study, we had carried out the ion chamber calibration through preliminary tests P1 and P2 respectively to ensure the feasibility of the following test cases. The AP-PA test was effectuated to obtain the conversion factor of the ion chamber. The primary tests presented a high confidence (the passing rate more than 99\%) in the reliability and efficiency of the dosimetry systems used in this study.

The plan dosimetry results for the five test cases shown in Table 2 indicate that our clinic has met the dose requirements specified in 0889 . We also calculated a ratio between our planning results and the benchmark values of TG 119. Different DX (D5, D10, D20, D30, D95, and D99) of IMRT and VMAT plans are comparable to AAPM TG 119 plans. Meanwhile, all results were similar to Kadam A et al.'s data. ${ }^{[17]}$ we both tested single energy (IMRT, 6 MV) and all criteria have been achieved by following TG119 protocol in our clinic..

It is necessary to evaluate the accuracy of the IMRT and VMAT system before performing clinical treatment ${ }^{[18,19]}$. TG 119 test suite is helpful in evaluating the commission of treatment planning and delivery. We set CL as a benchmark for commission and QA of IMRT and VMAT technics with different energy beams and the results help us gain confidence in the accuracy of the treatment. Based on these measurements above and the followed analyses, it is obvious that the CLs obtained in our institute are superior to the benchmark recommended by the TG 119. The mean CLs for this accelerator ranged from $0.40 \%$ to $3.76 \%$, in which the SMLC and VMAT were less than what the TG119 recommended (CLs of $4.7 \%$ ) for low dose region. Meanwhile, all other parameters have been achieved following TG119 protocol in our clinic. For the FF photo beams and FFF photo beams, the mean gamma passing rate with criteria of $3 \% / 3 \mathrm{~mm}$ were higher than 95.31\%. The CL of FF and FFF photon beams were less than 5.35\%, which were below 6.3. Kadam A. et al. ${ }^{[17]}$, Zhang et al. ${ }^{[13]}$ and Kaviarasu et al. ${ }^{[19]}$ obtained the same result as ours. The CL recommended by TG 119 was 12.4. While our $\gamma$ passing rates for test cases were lower than those of studies by Zhang et al. ${ }^{[13]}$ Our results were similar to Kadam and Sharma's data, ${ }^{[17]}$ and all of us tested single technology and single energy (IMRT, $6 \mathrm{MV}$ ). Kaviarasu et al. ${ }^{[18]}$ reported similar results to ours.

In addition, the plans depends on the experience of the planners to some extent. TG 119 has been presented as a practical tool to evaluate the quality of an IMRT system as a part of the commissioning process. Although its results cannot pinpoint the source of the error, the CL of TG 119 is expected to help physicists determine whether the system can be applied for clinical 
practice ${ }^{[20]}$.

Gurjar OP et al ${ }^{[22]}$.and Ravikumar B et al. ${ }^{[23]}$ pointed the point dose measurements use of heterogeneous phantom of the same density pattern as that of actual human body site should be done for verifying the dose calculated by the algorithm and the phantom in the same shape and size as that of actual human chest level will be the good phantom for more accurate dose verification. Therefore, the CIRS Thorax phantom was selected for further verifications. As seen in table 5, the measured point doses in different locations of the heterogeneous phantom were compared with the results from the two algorithms, i.e., Collapse Cone (CC) and Monte Carlo (MC). The MC algorithm, which simulates the photon and electron transport processes in different kind of materials, is potentially the most accurate method for the calculation of dose distributions ${ }^{[24]}$. As can be seen in table 5 , although it is not evident, most of the mean standard deviations from the MC algorithm were smaller than those from CC. These results from both CC and $\mathrm{MC}$ suggested that the two algorithms in the treatment planning system show similar accuracy for all test cases, almost all the relative difference between measurements and calculations were less than 3\% and these accuracies were believed to be enough for the clinical treatment. The accuracy of the MC algorithm could be seen from a simple square filed test case with film in the material possesses high density, i.e. the spine region $\left.{ }^{[25}\right]$. The dose distributions from the MC algorithm were generally 3\% 4\% higher than those from CC, and show good agreements with the actual measurements.

This article makes a comprehensive assessment for the performance of uRT-Linac 506C and its treatment planning system.. The deviation between measured point doses of Static IMRT, Dynamic IMRT and VMAT and planned doses were within 3.76\%. Measured film dosimetry gamma passing rates of Static IMRT, Dynamic IMRT and VMAT were $>95.31 \%$ and CL were within $5.35 \%$

\section{CONCLUSION}

We evaluated the planning and delivery accuracy using FF and FFF beams, as well as IMRT and RapidArc techniques, using ion chamber and EBT 3 films based on TG-119 protocol. Based on this analysis performed in line with the TG 119 recommendations, it is evident that the uRT treatment planning system and uRT-Linac 506C have commissioned Static IMRT, Dynamic IMRT and VMAT technics with adequate accuracy.

\section{Acknowledgements}

The authors thank the Department of Radiation and Medical Oncology, Zhongnan Hospital of Wuhan University. Thanks for all colleagues' help and guidance.

\section{Funding}

This project is financially supported by Zhongnan Hospital of Wuhan University Science, Technology and Innovation Seed Fund, Project znpy2019022

\section{Availability of data and material}

The datasets analyzed during the current study are available from the corresponding author by reasonable request 


\section{Authors' contributions}

XW and DJ contributed to conception and design of the study and drafted the manuscript; JS and XW participated in data collection and literature research; JZ, HZ, JS, DW, ZB and HL contributed to data analysis and interpretation. All authors read and approved the final manuscript

\section{Ethics approval and consent to participate}

Not applicable.

\section{Consent for publication}

Not applicable.

\section{Competing interests}

The authors declare that they have no competing interests

\section{Author details}

Department of Radiation and Medical Oncology, Hubei Key Laboratory of Tumor Biological Behaviors, Hubei Cancer Clinical Study Center, Zhongnan Hospital of Wuhan University, Wuhan, China, 430071.

\section{Reference}

1. Lambrecht M, Nevens D, Nuyts S. Intensity-modulated radiotherapy vs. parotid-sparing 3D conformal radiotherapy. Effect on outcome and toxicity in locally advanced head and neck cancer. Strahlenther Onkol. 2013;189(3):223-9.

2. Anand AK, Jain J, Negi PS, Chaudhoory AR, Sinha SN, Choudhury PS et al. Can dose reduction to one parotid gland prevent xerostomia? A feasibility study for locally advanced head and neck cancer patients treated with intensity modulated radiotherapy. Clin Oncol (R Coll Radiol). 2006;18(6):497-504.

3. Shaikh M , Burmeister J, Joiner M, Pandya S, Zhao B ,, Liu Q et al. Biological effect of different imrt delivery techniques: smlc, dmlc, and helical tomotherapy. Medical Physics, 2010. 37(2), 762-770.

4. Otto K. Volumetric modulated arc therapy: IMRT in a single gantry arc. Medical Physics, 2008, 35(1):310-317.

5. Watanabe Y. A Practical Guide to Intensity-Modulated Radiation Therapy. Journal of Applied Clinical Medical Physics,4,4, 2003, 30(9):2565.

6. Georg D. Guidelines for the verification of IMRT. Radiotherapy \& Oncology, 2011, 99(2):520-30.

7. Ling CC, Zhang P, Archambault Y, Bocanek J, Tang G, Losasso T.et al. Commissioning and quality assurance of RapidArc radiotherapy delivery system. Int J Radiat Oncol Biol Phys, 2008, 72(2):575-581.

8. Losasso T, Chui CS, Ling CC. Comprehensive quality assurance for the delivery of intensity modulated radiotherapy with a multileaf collimator used in the dynamic mode. Medical Physics, 2001, 28(11):2209.

9. Palta JR , Deye JA, Ibbott GS, Purdy JA. , \& Urie, MM. Credentialing of institutions for imrt in clinical trials. international journal of radiation oncology biology physics, 2004.59(4), 
1257-1259..

10. Ibbott GS, Followill, DS, Molineu HA, Lowenstein JR, Alvarez PE, Roll JE, et al. Challenges in credentialing institutions and participants in advanced technology multi-institutional clinical trials. Int J Radiat Oncol Biol Phys, 2008, 71(1):S71-S75.

11. Molineu A, Hernandez N, Nguyen T, Ibbott G \& Followill D. Credentialing results from IMRT irradiations of an anthropomorphic head and neck phantom. Medical Physics, 2013, 40(2):022101.

12. Ezzell GA, Burmeister JW, Dogan N, LoSasso TJ, Mechalakos JG, Mihailidis D et al. IMRT commissioning: multiple institution planning and dosimetry comparisons, a report from AAPM Task Group 119. Medical Physics, 2009, 36(11):5359-5373.

13. Zhang J,Jiang D,Liu H,Shen J, Wang D, Chen $\mathrm{C}$ et al. Analyzation of the local confidence limits for IMRT and VMAT based on AAPM TG119 report .Med Dosim, 2020, 45: 66-72.

14. Mynampati DK, Yaparpalvi R, Hong L, Hsiang CK,, Mah D,et al . Application of AAPM TG 119 to volumetric arc therapy (VMAT). Journal of Applied Clinical Medical Physics, 2012, 13(5):3382.

15. Nithya L, Arunai N R N, Rathinamuthu S \& Pandey MBl. Analyzing the performance of the planning system by use of AAPM TG 119 test cases. Radiological Physics \& Technology, 2016, 9(1):22-29.

16. Wen N, Zhao B, Kim J, Chin-Snyder K, Bellon M, Glide-Hurst C, et al.Imrt and rapidarc commissioning of a truebeam linear accelerator using tg-119 protocol cases. Journal of Applied Clinical Medical Physics, 2014. 15(5), 74-88.

17. Kadam AS, Sunil. Estimation of local confidence limit for $6 \mathrm{MV}$ photon beam IMRT system using AAPM TG 119 test protocol. International Journal of Cancer Therapy and Oncology. 2016.

18. LoSasso T, Chui CS, Ling CC. Comprehensive quality assurance for the delivery of intensity modulated radiotherapy with a multileaf collimator used in the dynamic mode.[J] .Med Phys, 2001, 28: 2209-19.

19. Kaviarasu K, Nambi Raj NA, Hamid M, Giri Babu AA, Sreenivas L, Murthy KK et al . Verification of Dosimetric Commissioning Accuracy of Intensity Modulated Radiation Therapy and Volumetric Modulated Arc Therapy Delivery using Task Group-119 Guidelines. J Med Phys, 2017, 42(4):258-265.

20. G ordon JD, Krafft SP, Jang S, Smithraymond L, Stevie MY \&Hamilton RJ et al. Confidence limit variation for a single IMRT system following the TG 119 protocol. Med Phys 2011, 38:1641-8;

21. Rangel A \& Dunscombe P. Tolerances on MLC leaf position accuracy for IMRT delivery with a dynamic MLC. Med Phys, 2009, 36(7), 3304-9.

22. Gurjar OP, Paliwal RK, Mishra SP.A Dosimetric Study on Slab-pinewood-slab Phantom for Developing the Heterogeneous Chest Phantom Mimicking Actual Human Chest. J Med Phys,2017. 42(2), 80-85.

23. Ravikumar B, Lakshminarayana S. Determination of the tissue inhomogeneity correction in high dose rate brachytherapy for iridium-192 source. J Med Phys. 2012;37:27-31

24. Reynaert N, Marck SCVD , Schaart DR, Zee WVD, Vliet-Vroegindeweij CV \& Tomsej M et al. Monte Carlo treatment planning for photon and electron beams[J]. Radiation Physics \& Chemistry, 2007, 76(4):643-686. 
25. Fotina I, Winkler P, Thomas Künzler, Reiterer J, Simmat I \& Georg D et al. Advanced kernel methods vs. Monte Carlo-based dose calculation for high energy photon beams[J]. Radiotherapy \& Oncology, 2009, 93(3):645-653. 
Figures

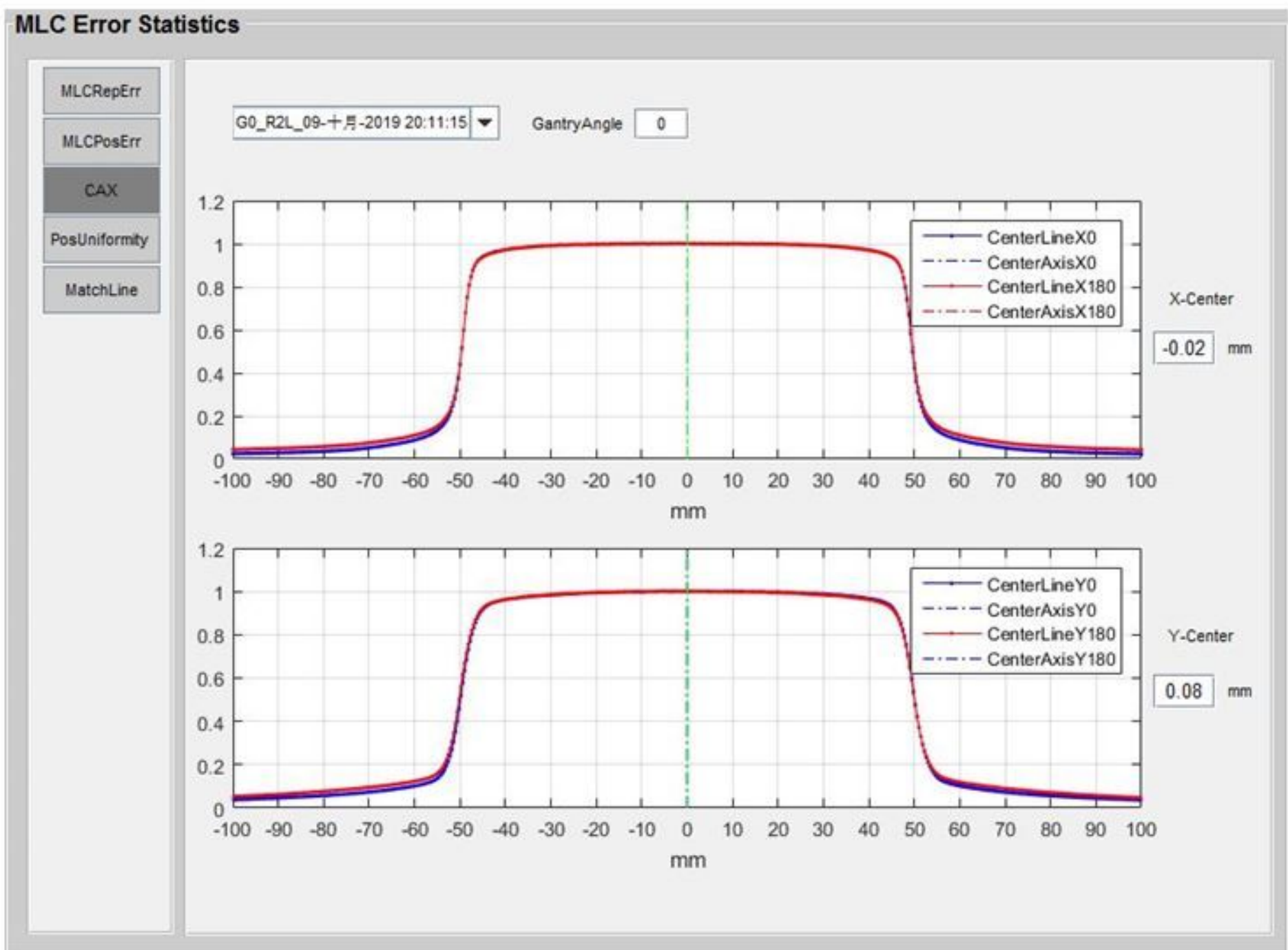

\section{Figure 1}

The linear accelerator of United Imaging Healthcare's CT linac uRT-Linac 506C 


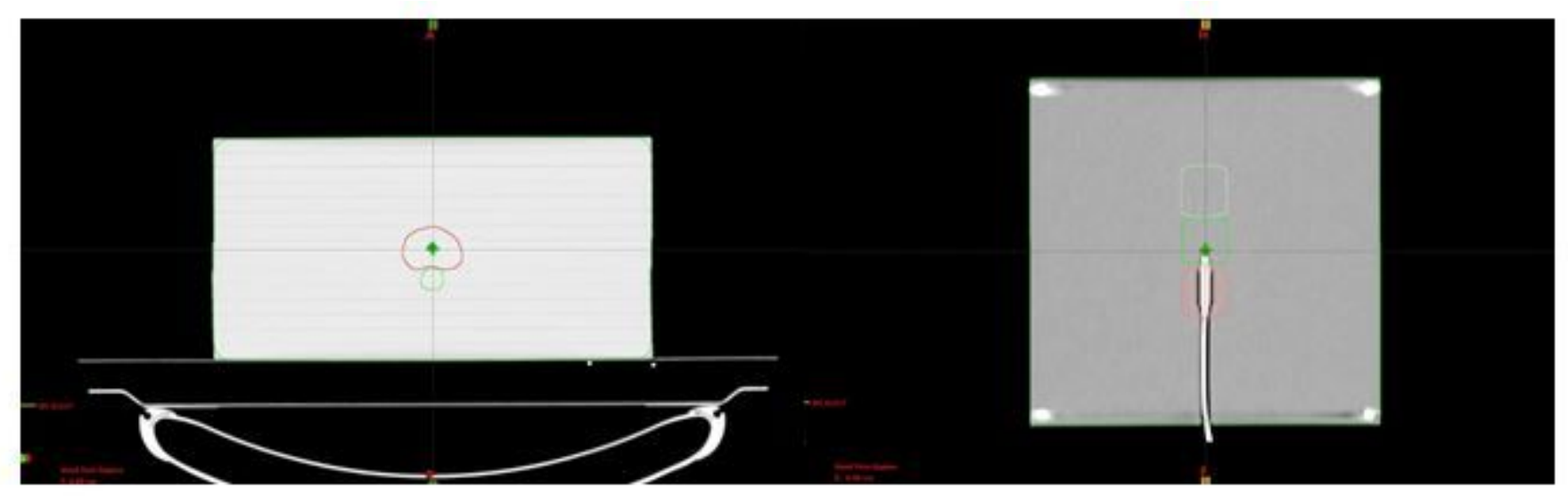

A

B

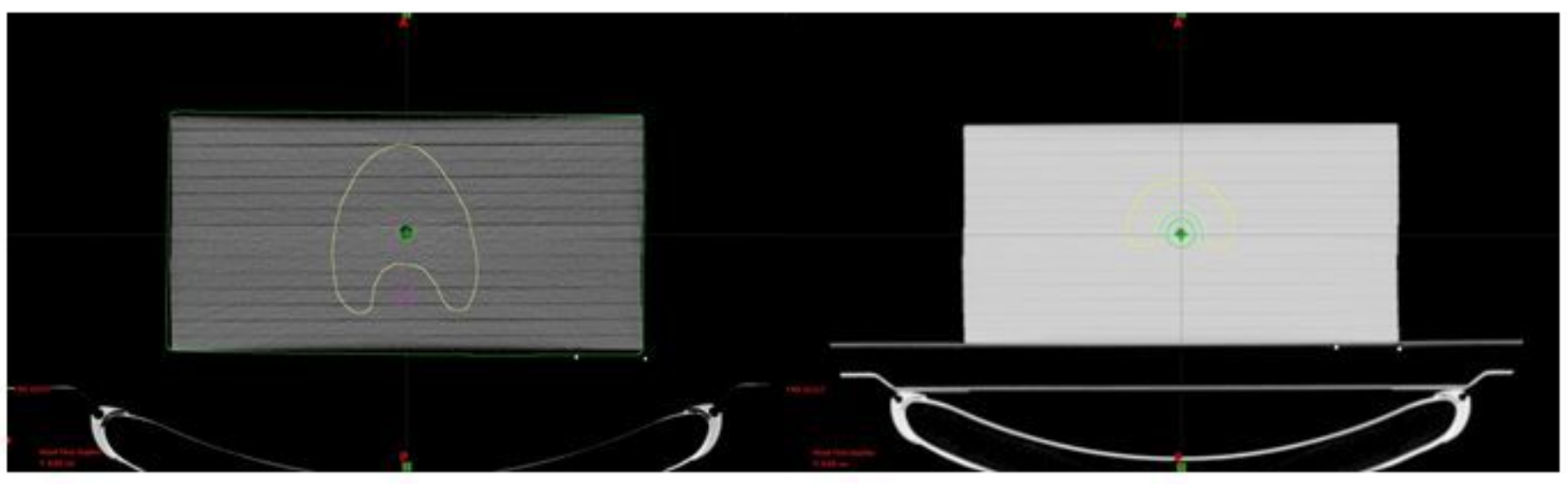

Figure 2

The test structures of Prostate (A), Multi Target (B), Head-and-neck (C) and C-shaped (D) by homogeneous phantom. 


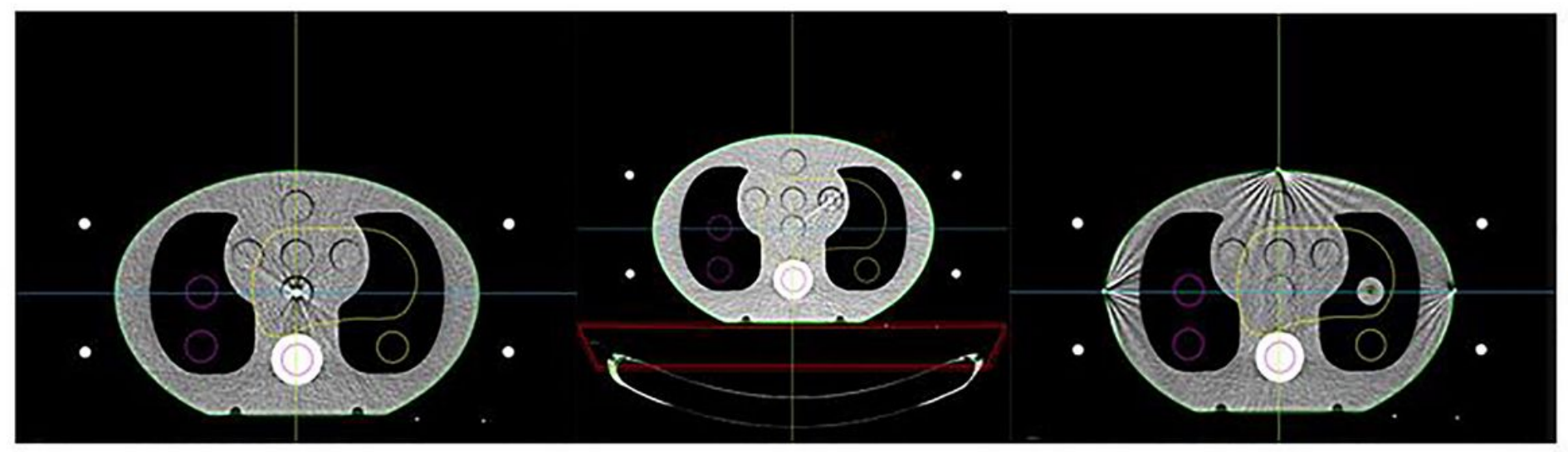

A

$\mathrm{B}$

$\mathrm{C}$

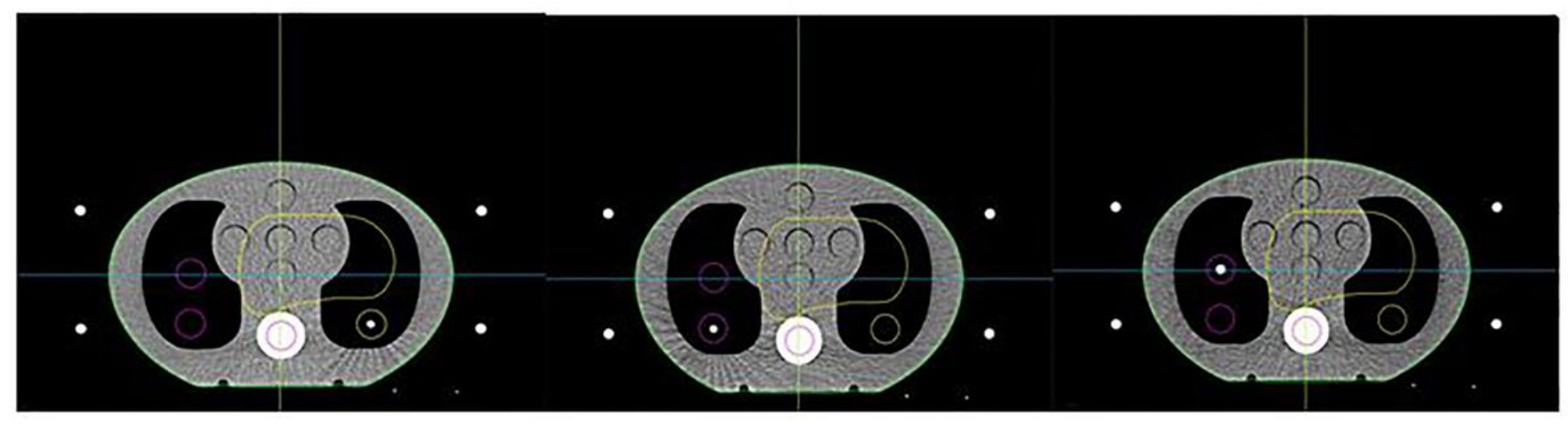

$\mathrm{C}$

$\mathrm{D}$

$\mathrm{E}$

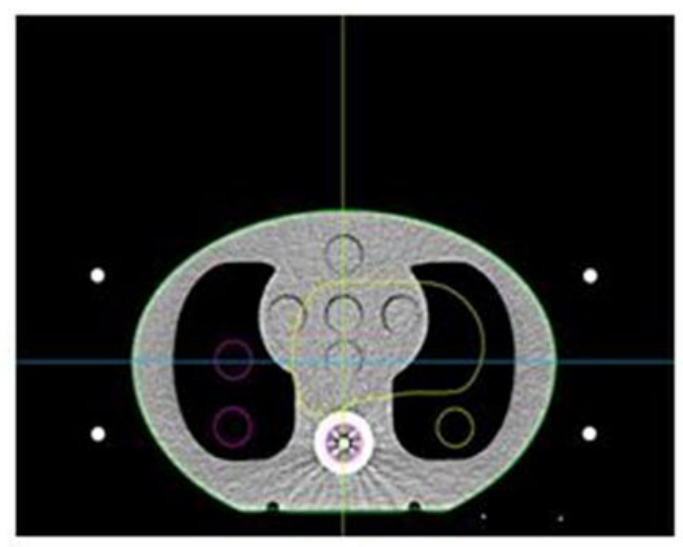

$\mathrm{F}$

Figure 3

The test structures of In PTV Down (A), In PTV Up (B), In PTV Lung (C), Out PTV Left (D), OUT PTV Right down (E), Out PTV Right Up (F) and In Spine (G) by heterogeneous chest phantom. 